\title{
Elisabeth, Ursule et les Onze mille Vierges : un cas d'invention de reliques à Cologne au XIle siècle
}

In: Médiévales, N²2-23, 1992. pp. 173-186.

Citer ce document / Cite this document :

Moulinier Laurence. Elisabeth, Ursule et les Onze mille Vierges : un cas d'invention de reliques à Cologne au XIle siècle. In: Médiévales, №22-23, 1992. pp. 173-186.

doi : $10.3406 /$ medi.1992.1247

http://www.persee.fr/web/revues/home/prescript/article/medi_0751-2708_1992_num_11_22_1247 
Médiévales 22-23, printemps 1992, pp. 173-186

Laurence MOULINIER

\section{ÉLISABETH, URSULE ET LES ONZE MILLE VIERGES : UN CAS D'INVENTION DE RELIQUES À COLOGNE AU XII' SIÈCLE}

De nombreux travaux ont montré que les reliques des saints exercèrent une fascination croissante sur le clergé et les fidèles au Moyen Age et que la vénération intense qui s'y attachait n'allait pas parfois sans quelques excès ; il est ainsi facile, nous dit André Vauchez, " d'ironiser sur les innombrables dents de saint Jean-Baptiste ou cheveux du Christ que les églises d'Occident se procurèrent, souvent à grand prix, auprès d'habiles imposteurs "'. Encore ne s'agit-il dans ces exemples que de reliques de saints " anciens ", dont la popularité n'empêcha pas de nouveaux cultes à caractère local de naître et de se développer, suite à la découverte, généralement fortuite, des restes de personnages plus proches dans le temps et dans l'espace. Une fois mis au jour, ces restes, pour la plupart anonymes, se voyaient attribuer une identité et une histoire; ainsi se rejoignaient, dans cette entreprise de "fabrique de saints " - pour reprendre un mot d'André Vauchez $^{2}$ - qui caractérisa une partie du Moyen Age occidental, les deux sens du mot inventio : trouver des ossements impliquait qu'on leur trouvât une histoire, l'invention de reliques stimulait l'inventivité des hommes. De cette coïncidence entre les deux significations du terme, un exemple particulièrement frappant nous est fourni par l'invention des reliques de "sainte Ursule " et des Onze mille vierges au XII' siècle, à Cologne : la découverte de leurs reliques supposées permit à leur histoire et à leur culte de dépasser rapidement les limites du cadre rhénan au point que le monastère des Onze mille vierges

1. La spiritualité du Moyen Age occidental, vII/-XI/·siècles, Paris, P.U.F., 1975, p. 149.

2. La sainteté en Occident aux derniers siècles du Moyen Age, Rome, École française de Rome, 1988, p. 152. 
de Cologne devint un des principaux lieux de pèlerinage de la chrétienté ${ }^{3}$. C'est donc à la légende d'Ursule et des Onze mille vierges que nous nous intéresserons ici, en tâchant d'analyser les rapports qui lient reliques et vie de saint, et de montrer que le hasard comme le génie humain eurent leur rôle à jouer dans la formation d'une telle légende qui, de locale, devint universelle.

Pour commencer, quelques faits :

$\mathrm{Au}$ IVe siècle de notre ère, plusieurs vierges furent martyrisées à Cologne, comme l'atteste une inscription que l'on peut y lire sur un mur du chœur de l'actuelle église Sainte-Ursule. Attribuée à un certain Clematius et datant sans doute de la seconde moitié du IVe siècle, elle nous apprend que notre homme, venu de l'Orient, aurait rebâti la basilique des vierges martyres en accomplissement d'un vœu ${ }^{4}$.

Au XII siècle, toujours à Cologne, la construction d'une nouvelle enceinte permit la mise au jour de nombreux ossements humains, aussitôt identifiés comme les restes de ces vierges anonymes dont seules dix lignes lapidaires prouvaient jusqu'alors le caractère historique. Par la suite, leur nombre et leur popularité allèrent croissant, en un mouvement qui culmina dans la seconde moitié du XII siècle, avec Élisabeth, mystique bénédictine du monastère de Schönau, à qui une série d'extases visionnaires révéla l'identité et l'histoire de ces vierges.

La légende des Onze mille vierges avait dès lors trouvé sa forme définitive, et on peut la résumer comme suit : aux premiers siècles de notre ère Ursule, fille du roi de Bretagne, met une condition à son mariage avec le fils du roi d'Angleterre : qu'on lui accorde un délai de trois ans, des vaisseaux et dix suivantes pour se rendre en pèlerinage à Rome et qu'on lui confie, à elle et aux autres, mille vierges. Le jeune homme, quant à lui, devait mettre à profit ce laps de temps pour se faire baptiser et instruire dans la foi. Ursule obtient satisfaction, s'embarque avec sa milice d'un nouveau genre et parvient à Rome, où le pape Cyriaque réserve un accueil enthousiaste à cette multitude de vierges. Sur le chemin du retour, la troupe virginale est massacrée par les Huns, qui assiégeaient alors Cologne.

La popularité de cette légende ne devait pas se démentir pendant tout le Moyen Age, si l'on en juge par le nombre des manuscrits qui nous l'ont transmise, le vaste trafic de reliques auquel elle donna lieu, et les remarquables fresques qu'elle inspira, au XVe siècle, à un Memling ou à un Carpaccios. Précisons tout de suite que c'est au sens

3. Au XIII siècle, Thomas de Cantimpré compte l'église de Cologne parmi les quatre églises saintes de la chrétienté, avec celles de Rome, de Jérusalem, et de Trèves (Bonum universale de apibus, I. II, cap. LIII, Douai, Éd. Baltazar Beller, 1627, p. 493).

4. Guy DE TERvarent, La légende de sainte Ursule dans la littérature et dans l'art du Moyen Age, Paris, 1931, p. 8.

5. La châsse peinte par Memling \& consacrée le 21 octobre 1489 se trouve actuellement au Memlingmuseum de Bruges, le cycle de Carpaccio, peint fin xve pour l'école Sainte-Ursule à Venise, y est aujourd'hui conservé à l'Accademia. 
moderne du terme que nous employons le mot "légende ", n'ayant pas de preuve que les personnages de cette histoire, Ursule, ses compagnes, ou même le pape Cyriaque aient existé ailleurs que dans l'imagination des hommes. Tout autre était, au Moyen Age, le sens de ce mot qui reçut ses lettres de noblesse avec Jacques de Voragine au XIII' siècle : pour lui qui fit une place à ces vierges dans sa Légende dorée comme pour d'autres auteurs, legenda désignait tout texte lu à l'église ou au réfectoire, et pouvait être synonyme de " récit de vie de saint ». Chez Sicard, évêque de Crémone au XII siècle, legendarius signifie ainsi " recueil de Vies de saints " ${ }^{6}$. Sans entrer ici dans une analyse détaillée du genre hagiographique et de sa réception au Moyen Age, disons simplement que pour le public de ces Vitae, la distinction entre vrai et fictif n'était pas pertinente : l'authenticité des événements décrits était indubitable pour l'homme médiéval qui y adhérait, y croyait comme à un témoignage immédiat. Aussi la structure de la notice que Jacques de Voragine consacre à ces vierges ne diffère-t-elle en rien de celles qu'il rédige sur d'autres saints, authentiques et authentifiés à nos yeux et proches de lui dans le temps comme dans la mémoire de ses contemporains, tels Bernard de Clairvaux ou François d'Assise : à l'énoncé d'une "vie " et des faits édifiants qui l'ont marquée, succède l'énumération des interventions miraculeuses du saint après sa mort. Une des vierges, dont le corps avait été donné à un abbé par "l'abbesse de Cologne " à condition de "le placer en son église dans une châsse d'argent ", se vengea ainsi de l'oublieux abbé en désertant la châsse de bois où il l'avait laissée : elle « descendit corporellement de dessus l'autel et après avoir fait une profonde révérence devant l'autel, elle passa, en présence de tous les moines effrayés, à travers le chœur et se retira ". L'abbé eut beau présenter ses excuses et s'engager à faire confectionner au plus tôt une châsse d'argent, il ne put récupérer le corps ni en obtenir un nouveau, nous dit Jacques de Voragine ${ }^{7}$.

On a reconnu là le type du miracle de punition, suivi, chez notre auteur, d'un miracle de guérison d'un genre particulier, dont on trouve un écho dans le Bonum universale de apibus ${ }^{8}$ de Thomas de Cantimpré : les vierges se manifestent, ici à un religieux, là à une sœur de l'hospice des pauvres de Bruxelles, tous deux gravement malades, et leur rendent la santé, le temps pour eux de proclamer la gloire des vierges colonaises, juste avant de rendre l'âme.

Le lecteur nous pardonnera cette présentation un peu longue, destinée d'une part à le familiariser avec une légende qui n'a guère plus cours et à montrer d'autre part qu'au XIII' siècle, qui vit fleurir les

6. Sicardus, Mitrale sive summa de officiis ecclesiasticis, 5, pr., PL 213, cité par A. BlaISE, Lexicon latinitatis medii aevi, CCCM, Turnhout, Brepols, 1986, p. 530. p. 298.

7. La légende dorée, trad. J.B.M. Roze, Paris, Garnier Flammarion, 1967, t. II,

8. Bonum universale de apibus, op. cit., 1. II, cap. LIII, p. 494. 
Vies de saints, les Onze mille vierges sont parfaitement intégrées dans le paysage hagiographique. Reste, pour nous, à nous interroger sur leurs avatars aux siècles précédents et à montrer que le XII siècle fut une étape décisive dans la formation de leur légende : quelles furent les autres étapes, et comment s'opéra la diffusion de la légende de sainte Ursule et de ses compagnes ? Quel rôle y joua, volontairement ou non, Élisabeth de Schönau, et qui était au juste celle que l'on tient pour une sainte en Allemagne mais dont le culte ne fut jamais reconnu et dont même l'inscription au Martyrologe romain en 1584, sous Grégoire XIII, contient une erreur sur sa personne ${ }^{9}$ ? Telles sont les questions auxquelles nous essaierons de répondre dans le présent exposé en racontant, pour ainsi dire, l'histoire de leur histoire car, si l'existence des saintes fêtées le 21 octobre est particulièrement douteuse, la genèse, l'évolution et l'importance de ces personnages au Moyen Age méritent qu'on s'y arrête.

Les vierges martyrisées à Cologne au Bas-Empire furent à peu près oubliées pendant 500 ans : seule en gardait le souvenir une inscription latine gravée sur la pierre d'une église et il faut attendre le IX' siècle pour retrouver leur trace, dans les textes cette fois. Martyrologes, calendriers et litanies nous apprennent que leur culte fut dès lors célébré à Cologne, où l'église restaurée par Clématius existait toujours et abritait une communauté de femmes. Les textes les plus anciens ne nomment pas ces vierges, mais un de leurs caractères s'affirme : elles étaient très nombreuses, voire innombrables. C'est ainsi qu'en 848, dans son Martyrologe en vers, Wandalbert de Prüm évoque des "milliers de vierges" à la date du 21 octobre $^{10}$ et que deux calendriers peu postérieurs, l'un sans doute de Herford, l'autre d'Essen, annoncent : " À Cologne 11000 saintes vierges ».

Un siècle plus tard, au $X^{\circ}$ siècle, onze noms apparaissent : Martha, Saula, Brictola ou Britola, Gregoria, Saturnina, Sabatia ou Rabacia, Pinnosa ou Vinnosa, Ursula ou Ursola, Sentia, Palladia et Saturia. À ce stade de son développement, la légende primitive d'Ursule et de sa suite diffère grandement de celle que les derniers siècles du Moyen Age nous ont léguée : Ursule ne figure pas à la tête des vierges, et leur nombre ne semble pas arrêté. Onze ou onze mille ? $\mathrm{W}$. Levison a montré que cette hésitation si surprenante pouvait s'expliquer par le signe « $\overline{\mathrm{XI}}$ » auquel le tiret conférait dans les manus-

9. Les Bollandistes la considèrent comme sainte et la fêtent le 18 juin, mais le Martyrologe romain la dit cistercienne et son nom y figure sans mention des visions. Cf. Charles Baumgartner éd., Dictionnaire de spiritualité ascétique et mystique, fasc. XV, Paris, Beauchesne, 1958, col. 587. Quant à la Légende dorée, elle évoque longuement (op. cit., t. II, pp. 348-367) une sainte homonyme postérieure, Élisabeth de Hongrie, morte en 1231, mais ne cite pas une seule fois Elisabeth de Schönau.

10. Cité par Guy de Tervarent, op. cit., p. 11 : "Millia mactavit ductricibus inclita sanctis ». 
crits l'une ou l'autre valeur'". Quoi qu'il en soit, c'est l'idée d'une foule de vierges, d'une véritable armée virginale qui devait l'emporter définitivement. Dans un sermon du $X^{e}$ siècle connu comme le Sermo in natali, prononcé à Cologne un 21 octobre devant la communauté religieuse vouée au culte de ces vierges, l'auteur forme le vœu que leur histoire trouve un écrivain pour la conter ${ }^{12}-$ ce qui permet de dater ce texte d'avant les années 969-976, durant lesquelles vit le jour, comme en réponse à ce vœu, la première passio consacrée à ces vierges, la passion "Fuit tempore pervetusto »; les vierges y apparaissent comme une valeureuse armée céleste, dont il faudra léguer à la postérité le récit des combats. "Cur caelestis huius exercitus a principio gesta simul et proelia non scriberentur ", demande l'auteur ; son appel sera entendu, et l'armée dotée d'un chef, Ursule, que l'on voit se glisser tardivement parmi ses compagnes dans les textes, mais y prendre rapidement leur tête. L'entrée d'Ursule dans le groupe des vierges peut pour sa part s'expliquer par la découverte, au $\mathrm{X}^{\mathrm{e}}$ siècle, d'une autre inscription lapidaire dans l'église des vierges saintes de Cologne, l'actuelle église Sainte-Ursule où Klinkenberg la redécouvrit à son tour sous une couche de chaux, en $1893^{13}$. Ce qu'on pouvait $\mathrm{y}$ lire au $\mathrm{X}^{\mathrm{e}}$ siècle est indiqué en majuscules dans les lignes suivantes où les lacunes ont été comblées :

In hoc tum ULO INNOCIS VIRGO JACET

no MINE URSULA VIXIT

a NNIBUS OCTO

$m$ ENSIBUS DUOBUS

d IENS (sic) QUATTOR

Dans cette inscription funéraire concernant une fillette de huit ans, on ne voulut voir qu'une qualité, "vierge innocente ", et qu'un nom, "Ursule ", qu'on ajouta à ceux des vierges dont l'église pouvait se prévaloir de posséder des reliques ; l'auteur anonyme de la passion "Fuit tempore... " témoigne en effet de leur existence en déclarant, dans son prologue, écrire à la requête d'une communauté de femmes dont l'église abrite des restes de vierges, " in qua virginum tumulata sunt pignora ${ }^{14}$.

Venons-en maintenant à l'élection d'Ursule à la tête des Onze mille vierges : c'est paradoxalement à l'existence de reliques d'autres vierges que le personnage d'Ursule doit son titre de reine incontestée de la troupe virginale. En effet c'est après le transfert à une communauté d'Essen, vers 950 , des reliques de celle que l'on vénérait comme sainte Pinnosa, que les religieuses de l'église des vierges saintes de

11. W. Levison, Das Werden der Ursula Legende, Cologne, 1928, pp. 39-42.

12. GuY DE Tervarent, op. cit., p. 9.

13. Vie des saints et des bienheureux selon l'ordre du calendrier par les RR.PP. bénédictins de Paris, Oct., tome X, Paris, 1952, p. 677.

14. W. Levison, op. cit., p. 36. 
Cologne, conscientes du risque de concurrence, avaient demandé à un homme - dont nous ne connaissons que l'initiale « $H$ » - un récit qui mît en avant cette Ursule dont on avait découvert le tombeau dans leur église ; ainsi était née la passion "Fuit tempore pervetusto » adressée à Gero, archevêque de Cologne de 969 à 976, à la fois, pour Guy de Tervarent ${ }^{15}$, "cuvre de propagande " et " plaidoyer pro domo » où le récit de la sépulture des Onze mille vierges est suivi de l'épigraphe de Clematius, dans le but d'authentifier les reliques. Seuls six manuscrits de cette ouvre nous sont parvenus mais au $\mathrm{XI}^{\mathrm{e}}$ siècle, elle servit de trame à une nouvelle passion, "Regnante domino ", dont $\mathrm{W}$. Levison a recensé une centaine de manuscrits attestant le succès ${ }^{16}$.

À cette époque, l'histoire des vierges venues d'Angleterre était en place dans ses grandes lignes et on distinguait même, dans la troupe édifiante, des personnalités et un chef, mais le décalage était encore grand entre les dimensions ambitieuses de l'histoire et le volume de ses preuves, des signes tangibles susceptibles d'asseoir définitivement sa crédibilité et, partant, sa diffusion. Si l'on osait un jeu de mots, on pourrait dire que c'est peut-être faute d'ossements que l'histoire des Onze mille vierges manquait encore un peu de corps.

La révolte d'Henri $V$ contre son père Henri IV fut donc en un sens providentielle : poursuivi par son fils, Henri IV se réfugia en 1106 à Cologne, où il ordonna, pour la défense de la place, la construction de nouveaux remparts qu'au reste l'extension de la ville exigeait, et qui embrassaient pour la première fois plusieurs faubourgs, notamment celui où se trouvait l'église des vierges saintes ${ }^{17}$. C'est là qu'en creusant, on tomba par hasard sur un grand nombre d'ossements. C'était l'emplacement d'une ancienne nécropole romaine : on y vit les restes des Onze mille vierges. La nouvelle de la découverte se répandit dans l'ensemble de la chrétienté et les reliques supposées furent aussitôt l'objet de translations, voire d'exportations dès 1113 .

Des problèmes tout à fait nouveaux surgirent alors : on avait soudain beaucoup plus de corps que de noms, dont les deux versions de la " passion » ne fournissaient qu'un petit nombre ; et comment justifier la présence de squelettes masculins parmi les ossements ? On dut improviser, mais chaque problème eut sa solution : on se mit à graver des pierres tombales, à imaginer des tituli pour les compagnes supposées d'Ursule et à leur inventer des parents puis, pour justifier l'importance des restes masculins, toute une escorte de rois, pape, cardinaux, évêques qui aurait partagé leur sort ; on trouva même le fiancé d'Ursule, en détournant la véritable épitaphe d'un vrai chrétien des premiers siècles : "Hic jacet in terris Etherius. Qui vixit annos viginti

15. G. DE TERVARENT, op. cit., p. 18.

16. Cf. W. Levison, op. cit., pp. 91-96.

17. Vie des saints et des bienheureux..., op. cit., p. 683. 
quinque fideles. In pace recessit. " L'âge correspondait parfaitement, le nom était plausible, on fiança donc Ursule et Etherius et l'abbaye de Deutz garda la pierre tombale ${ }^{18}$.

On pouvait faire dire bien des choses aux inscriptions lapidaires - le personnage d'Ursule devait sa popularité à l'une d'entre elles mais jusqu'où ? L'invention de la tombe d'Etherius suscita des questions auxquelles on ne sut pas d'abord répondre : que faisait par exemple à Cologne le fiancé censé attendre, d'après le récit qu'on en connaissait, le retour d'Ursule outre-Manche ? En 1156, l'abbé de Deutz, Gerlac, trouva une solution en décidant de recourir aux lumières d'Élisabeth, moniale de Schönau connue pour les visions qui lui apparaissaient régulièrement depuis 1152 .

Élisabeth était jeune encore, mais déjà célèbre. Née en 1129 en Rhénanie, elle avait été confiée à douze ans au monastère double de Schönau, où elle avait pris le voile en 1147 . De constitution très fragile, elle avait commencé à connaître l'extase et l'état de visionnaire après une sérieuse maladie en 1152 . Ses premières visions s'inscrivaient étroitement dans le cadre de l'année liturgique : outre le Christ et la Vierge, elle voyait en général les saints du jour ${ }^{19}$ et conversait avec eux. De ses saintes conversations son frère Egbert, alors chanoine à Bonn, lui demandait des récits détaillés : Élisabeth lui envoyait ses notes, qu'elle conservait dans un libellum caché sous sa couche d'après son propre témoignage - "Tunc significavi magistrae, ut afferret ad me libellum quemdam, quem in stratu meo absconderam, continentem ex parte ea quae fecerat Dominus mecum" - ${ }^{20}$, et qu'il mettait en forme. L'influence qu'il exerçait sur sa sœur et leur « collaboration " s'intensifièrent avec l'entrée d'Egbert à Schönau en 1155. Il faut croire qu'il sut intéresser aux visions de sa sœur un cercle de plus en plus large, car les visions se firent plus nombreuses et plus importantes, tendant de plus en plus à apporter une réponse à des questions concrètes sur la théologie, ou même à des cas de conscience, venant de toutes parts. Le succès grandissant de ses révélations, qu'Egbert consignait aussitôt par écrit, n'empêcha pas Élisabeth de traverser de graves crises de doute et d'accablement dont la Vita sanctae Elisabeth, composée par son frère, et la correspondance de la moniale - une vingtaine de lettres nous sont parvenues - nous renvoient l'écho.

Élisabeth correspondit entre autres avec la grande abbesse de Bingen, sainte Hildegarde (1098-1179) auprès de qui elle chercha réconfort et consolation, se plaignant de la méchanceté du monde, des moqueries que lui valaient ses visions et même des faussaires faisant

18. G. DE Tervarent, op. cit., p. 23.

19. Cf. Enciclopedia cattolica, Città del Vaticano, 1950, tome EA-GEN, p. 250.

20. Vita sancte Elisabeth, 4, in Beati Aelredi opera, PL 195, col. 121 : " Je fis signe alors à ma supérieure de $\mathrm{m}$ 'apporter un petit livre que j'avais caché dans ma couche et qui contenait pour une part ce que le Seigneur avait fait avec moi." " 
circuler sous son nom des oracles qu'elle n'aurait jamais osé rendre : "Audio et quosdam, litteras de suo spiritu scriptas sub nomine meo circumferre : de die judicii me prophetasse diffamaverunt, quod certe numquam facere praesumpsi, cum omnium mortalium cognitionem fugiat ejus adventus » (J'entends dire que certains font circuler sous mon nom des lettres de leur cru qui me diffament ; j'aurais, selon eux, prophétisé l'arrivée du jugement dernier, ce que je n'ai certes jamais eu l'audace de faire, puisque ce jour échappe à la connaissance humaine.), se lamente-t-elle ainsi dans une lettre éditée dans la Patrologie latine ${ }^{21}$ où elle apparaît aux prises avec deux sortes de peur, peur du monde et de ses railleries, peur de Dieu et de ses châtiments. En effet l'ange du Seigneur qui visitait Élisabeth dès qu'elle entrait en extase - c'est-à-dire, dans le texte latin, dès qu'elle sortait de son esprit, " mentis excessu " - ne ménageait pas la visionnaire si elle tardait à accomplir sa mission ; écrasée par la peur d'être taxée de fabulation, de passer pour une "auctrix novitatum " 22 selon ses propres termes, à une époque dominée par le culte des "autorités", où il n'était pas bon de mettre son originalité en valeur, Élisabeth n'osait parler, ce que l'ange du Seigneur lui reprocha parfois énergiquement : "Pourquoi caches-tu l'or dans la boue ? " lui demande-t-il ainsi avant de la fouetter avec colère à cinq reprises, si durement, affirme Élisabeth, qu'elle en souffrit dans tout son corps trois jours durant ${ }^{23}$.

Dans les déclarations de la mystique de Schönau il faut bien sûr faire la part du topos de l'ignorance prophétique : comme le prophète Jérémie et comme sa contemporaine Hildegarde de Bingen, Elisabeth se sent accablée par le poids de son ignorance, thème que l'auteur de sa Vita ne manque pas de développer en la présentant comme ignorant le latin et n'ayant appris des hommes que l'art de psalmodier ${ }^{24}$. C'est néanmoins à un point extrême qu'Élisabeth pousse l'autodénigrement, qui va de pair avec la rude ascèse qu'elle inflige à un corps valétudinaire. N'évoque-t-elle pas sa faible santé comme un « martyre intolérable " qu'elle supporte depuis sa jeunesse ? Cette langueur est source pour elle non seulement de souffrances physiques mais aussi de tortures mentales car son caractère inquiet à l'excès y voit un argument possible pour ses détracteurs et elle fait part à sa supérieure de cette nouvelle crainte : « J'ai peur, dit-elle, que mes maladies ne soient

21. Sancte Hildegardis opera, Ep. XLV Elisabethae magistrae in Schonaugia, PL 197, col. 215 B.

22. Ibidem : " ut arrogantiam evitarem et ne auctrix novitatum viderer, inquantum potui, omnia haec studui occultare " (J'ai pris soin, autant qu'il était en moi, de cacher tout cela, pour éviter de passer pour une arrogante et une fabulatrice.)

23. Ibidem, col. 215 C: "astitit mihi angelus Domini, dicens : Quare abscondis aurum in luto ? (...) Et hoc dicto elevavit super me flagellum, quod quasi in ira magna quinquies mihi amarissime inflixit; ita ut per triduum in toto corpore meo ex illa percussione languerem."

24. La Vita sancte Elisabeth, loc. cit., col. 120, la présente comme " interno spiritus sancti magisterio edocta » (instruite par l'enseignement intérieur de l'Esprit Saint). 
un scandale aux yeux des hommes et qu'ils ne pensent que c'est pour avoir très gravement péché que je suis la proie de tels tourments (Metuo, Domina, ne forte scandalizentur homines in infirmitatibus meis, et aestiment pro aliquibus gravioribus peccatis me ita torqueri) ${ }^{25}$."

Dans les réponses qu'elle adresse à Élisabeth, Hildegarde tente de la consoler mais surtout de l'exhorter à la mesure, à une discretio " mère de toutes les vertus sur la terre comme au ciel " que la jeune moniale néglige par trop : c'est, d'après l'abbesse de Bingen, « l'oiseau noir, c'est-à-dire le diable " qui pousse l'homme à des jeûnes, à une abstinence, bref à des mortifications extrêmes qui ne sont qu'un autre visage de l'orgueil ${ }^{26}$. Hildegarde ne parvint probablement pas à corriger le caractère exagérément sensible et excitable d'Élisabeth mais leurs œuvres respectives portent la trace d'une influence mutuelle. Encore faut-il préciser qu'Élisabeth n'est pas réellement l'auteur de ses œuvres: dans le tandem littéraire qu'elle forme avec son frère, c'est Egbert qui met en forme des révélations que sa sœur lui fournit pour ainsi dire à l'état brut, partie en allemand partie en latin. Rien à voir en tout cas dans cette collaboration avec le couple que formaient, à la même époque, Hildegarde de Bingen et son cher moine Volmar dont le rôle devait se borner à assister moralement la sainte et à assurer la correction grammaticale de ses écrits. Élisabeth est pour sa part si peu l'auteur de ses propres œuvres que dans certains des 150 manuscrits que nous en conservons encore aujourd'hui, les Revelationes beate Elisabeth portent le nom d'Élisabeth de Hongrie ${ }^{27}$. Le titre du Liber viarum Dei attribué à notre Élisabeth apparaît en tout cas comme calqué sur celui du Scivias que Hildegarde acheva en 1151 et, dans une des visions contenues dans ce "Livre des voies de Dieu » très diffusé au Moyen Age, Hildegarde est citée comme le modèle à imiter. Dans la troisième vision du livre, l'ange instructeur se manifeste ainsi à Élisabeth et lui montre un grand tas de livres, en disant : "Tous ces livres te seront dictés avant le jour du Jugement dernier. " Brandissant l'un d'entre eux, il ajoute : «Voici le Livre des voies de Dieu, qui sera révélé à travers toi quand tu auras vu et entendu ta sœur Hildegarde ${ }^{28}$. " Les deux femmes se virent-elles ? Nous l'ignorons. Toujours est-il que quand Hildegarde intervient dans la lutte contre les Cathares, Egbert n'est pas en reste et compose ses XIII Sermones contra Catharos ${ }^{29}$ et qu'à l'inverse, les révélations d'Élisa-

25. Ibidem, col. $182 \mathrm{C}$.

26. Lettre éditée par H. Omont, Notices et extraits des manuscrits de la Bibliothèque Nationale et d'autres bibliothèques, t. 38, Paris, 1903, p. 369.

27. Voir entre autres à la Bibliothèque Nationale les manuscrits français 1801, 1805, 1806 et 24868 et les commentaires de Kurt Köster, « Das visionäre Werk Elisabeth von Schönau ", Archiv für mittelrheinische Kirchengeschichte, 1952, pp. 79-119.

28. Liber trium virorum et trium spiritualium viriginum, Paris, 1513, p. 130.

29. XIII Sermones contra Catharas, PL 195, col. 14-98. L'hypothèse selon laquelle la légende d'Ursule aurait été créée pour lutter contre les Cathares en Rhénanie est écartée par Roth. 
beth sur les Onze mille vierges ne semblent pas étrangères aux compositions lyriques que ces saintes inspirèrent à Hildegarde. Ces vers de sa séquence $n^{\circ} 54$ "Aux Onze mille vierges »- « Quand Ursule eut ainsi parlé/Cette nouvelle se répandit parmi tous les peuples./Et ils dirent : " L'ignorance de la jeune fille, dans son innocence, $/ \mathrm{Ne}$ sait pas ce qu'elle dit $^{30}$. " - ne pourraient-ils d'ailleurs pas s'appliquer aussi à Élisabeth, en butte à l'incrédulité et à l'ironie de certains ?

Il est sûr en tout cas qu'en ce milieu du XII' siècle et dans cette partie de l'Empire, les Onze mille vierges et leurs reliques occupent largement les esprits : la présence d'un de ces précieux restes au Disibodenberg, monastère dont Hildegarde était magistra depuis 1136, est attestée en $1143^{31}$, de même qu'à Spanheim, autre monastère bénédictin du diocèse de Mayence. Quant à Trèves et à son diocèse, on n'y compte pas moins de dix emplacements de reliques, dont certaines se trouvent à la cathédrale de Trèves en 1124, à l'abbaye de SaintMatthias en 1148 et au monastère de Schönau en 1156 selon Guy de Tervarent ${ }^{32}$. Cette dernière date peut sembler tardive par rapport au début des fouilles de Cologne, un demi-siècle plus tôt ; mais il faut bien voir, d'une part, que l'exploitation de la nécropole de Cologne est très loin d'être terminée (en 1182 le monastère voisin d'Aldenberg reçut plus de mille corps ${ }^{33}$ et le volume des translations augmenta encore par la suite), d'autre part que l'abbaye de Schönau, fondée en $1130^{34}$, est encore, à l'époque qui nous intéresse, une abbaye nouvelle à qui ces fouilles viennent offrir ce qui lui manquait : un objet de vénération, gage d'un surcroît de prestige pour ce monastère qui pouvait déjà se prévaloir d'abriter une visionnaire.

D'après le premier chapitre du Liber revelationum Elisabeth de sacro exercitu virginum coloniensium ${ }^{35}$, c'est donc en 1156, " alors que Frédéric était empereur et Arnold II évêque de Cologne " qu'Hildelin, abbé de Schönau, organisa le transfert à son monastère du corps d'une de ces martyres, sur la tombe de laquelle on lisait les mots "Sancta Verena virgo et martir ". Ce corps lui avait été donné, ainsi qu'un autre dont on ignorait l'identité, par « G. abbé de Deutz ». Avant même l'arrivée des reliques au monastère, Élisabeth eut une extase et vit, sur la route qu'empruntaient les saints ossements, une flamme étincelante en forme de globe, que précédait un ange d'une très grande beauté, tenant d'une main un encensoir fumant et de l'autre un cierge ardent. Une seconde vision la prit pendant une messe

30. Hildegarde DE BIngen, Louanges, trad. L. Moulinier, Paris, La Différence, 1990 , p. 85.

31. Cf. les "Annales sancti Disibodi”, MGH, SS, XVII, p. 26.

32. G. DE TeRVARENT, op. cit., pp. 41-42.

33. Idem, op. cit., p. 21.

34. Ibidem, p. 44.

35. Édité par F.W.E. Roth, Die Visionen und Briefe der heiligen Elisabeth, Brünn, 1884, pp. $123-138$. 
qu'on célébrait en l'honneur de la vierge martyre : dans une clarté céleste, une vierge lui apparut, avec pour attributs une couronne et une palme. Élisabeth lui adressa alors la parole, lui demandant si "Verena » était réellement son nom, et quel était celui du martyr dont les restes étaient parvenus à Schönau. Verena, puisque c'était elle, lui révéla l'identité de Césarius, qui apparut à son tour à Élisabeth et lui raconta son histoire lors d'une nouvelle vision ${ }^{36}$.

Le dialogue était noué et Élisabeth, "un an durant " d'après ses propres termes, reçut ainsi de ses visions de "sainte Verena " des réponses précises aux questions les plus variées concernant les corps déjà découverts et ceux que l'on continuait d'exhumer. En fait, c'est sans doute jusqu'à sa mort, en 1164, que la moniale tâcha d'éclairer de ses "lumières " les zones d'ombre d'une histoire de plus en plus compliquée, si l'on en croit $\mathrm{Ph}$. Schmitz qui ne situe pas plus précisément qu'entre 1156 et 1164 la lettre inédite d'Elisabeth qu'il publie $^{37}$ : l'abbaye d'Odenheim, dans le diocèse de Spire, avait reçu elle aussi le corps anonyme d'une des Onze mille vierges de Cologne ; mais comment rendre un culte à une sainte dont on ignorait la vie, l'origine, et jusqu'au nom ? Dans sa réponse, Élisabeth identifia une sainte de plus et fournit même une courte biographie de celle qui selon elle s'appelait Vivencia, dont le père avait nom Arrianus et la mère Laetitia.

Nous ignorons en revanche de qui émanaient les questions concernant les Onze mille vierges auxquelles Élisabeth se charge de répondre dans son Liber Revelationum : elle dit parler à la demande " dudit abbé de la dite ville » - nous savons qu'il s'agit de Gerlac - qui soupçonnait les " inventeurs de corps saints " ${ }^{38}$ d'avoir fait fabriquer des tituli, dans un but frauduleux et lucratif. En effet les reliques se payaient, et même parfois fort cher, dans la mesure où elles étaient gage de pèlerinages, et donc de rentrées d'argent ; mais pour qu'elles aient un prix, là encore il leur fallait un nom...

Que les révélations d'Élisabeth, en garantissant l'authenticité de plusieurs de ces tituli, viennent en fait au secours d'un Gerlac mêlé de trop près aux fouilles en cours pour paraître honnête aux yeux de certains - Césaire d'Heisterbach, dans son Dialogue des Miracles $^{39}$, dénoncera l'intelligence entre clergé et faussaires, évoquant un certain Ulric, " habile à déterrer les reliques et dont le clergé de Cologne se servait beaucoup pour fouiller et mettre au jour les restes saints " - c'est ce que nous pouvons supposer sans l'affirmer :

36. F.W.E. Rorh, op. cit., pp. 123-124.

37. " "Visions" inédites de sainte Élisabeth de Schönau ", Revue bénédictine, XLVII, 1935, pp. 181-183.

38. F.W.E. RorH, op. cit., pp. 124-125: "Habebat quippe suspicionem de inventoribus sanctorum corporum, ne forte lucrandi causa titulos illos dolose conscribi fecissent"'.

39. Livre 8, chapitre 5 ; cité par G. De Tervarent, op. cit., p. 55. 
André Vauchez voit dans la fréquence des "inventions " de corps saints par le clergé depuis le IVe siècle une tentative, de la part de la hiérarchie ecclésiastique, d'orientation, et donc de contrôle de la dévotion populaire ${ }^{40}$. Il est clair en tout cas que la démarche d'Élisabeth n'a rien de spontané ou d'autonome et qu'on sent derrière ses "Révélations ", dès leur premier chapitre, la main de plusieurs « hommes de bonne réputation " qui ne lui ont pas permis de se taire et l'ont poussée, par leurs demandes insistantes, à ces recherches auxquelles elle-même rechignait, sachant trop bien que ses adversaires, " ceux qui combattent la grâce de Dieu en elle », allaient saisir l'occasion pour la "flageller de leurs langues", expression dans laquelle on reconnaît une thématique du fouet chère à Élisabeth ${ }^{41}$. Pourtant ni elle ni son frère ne sont à considérer comme des faussaires : ils croyaient sans doute sincèrement, par ces révélations, servir la cause de l'Église et de leur monastère. Malgré ces précautions oratoires, elle se plie donc au jeu des questions et des réponses et explique l'une après l'autre les inscriptions qu'on lui avait transmises pour examen ; nous n'en ferons pas autant et nous nous contenterons de deux exemples. Le titulus le plus remarquable à ses yeux est celui d'un pape : Sanctus Ciriacus papa Romanus, qui cum gaudio suscepit sanctas virgines et cum eis Coloniam reversus martirium suscepit. A la question de savoir pourquoi ce Cyriaque ne figurait pas sur la liste des papes et donc s'il avait réellement existé, elle répond, instruite par sainte Verena, que "le clergé de Rome ", indigné de voir le pape quitter le siège pontifical pour suivre ces vierges colonaises, s'était vengé de lui en le rayant de la liste des pontifes ${ }^{42}$. L'épitaphe d'Étherius suscitait elle aussi, nous l'avons vu, la perplexité : pouvait-on dire de ce païen qu'il avait vécu " annos viginti quinque fideles »? Pouvait-on justifier sa présence à Cologne alors que sa fiancée Ursule avait tenu à se séparer de lui pendant les trois ans de son pèlerinage ? La première de ces questions est aisée à résoudre : Étherius était si pur dans son cœur que même avant son baptême il vivait en chrétien, dans la foi, " fidelis ». Le second problème est plus complexe, et c'est l'ange du Seigneur qui se charge d'en donner la solution : le père d'Étherius, qui avait nom Agrippinus, était mort l'année du baptême de son fils, laissant une veuve, Demetria - selon l'épitaphe de la pierre voisine de celle d'Étherius, "Demetria regina », que venait d'élucider Verena qu'une vision divine enjoignit à Étherius de faire baptiser à son tour. La même vision lui apprit qu'il quitterait sa patrie pour aller à la rencontre de sa fiancée déjà sur le chemin du retour, et qu'ensemble ils subiraient le martyre dans la ville de Cologne. Étherius obéit et

40. La sainteté en Occident aux derniers siècles du Moyen Age, op. cit., p. 18.

41. F.W.E. Roth, op. cit., p. 123 : "De his enim me silere non permittunt quidam bone opinionis viri (...). Scio quidem, quoniam et hinc sumpturi sunt occasionem flagellandi me linguis suis hi qui adversantur gratie dei in me (...)."

42. F.W.E. Roth, op. cit., pp. 126-127. 
convainquit sa mère, qu'il emmena avec lui ainsi que sa jeune sœur identifiée d'après une autre épigraphe voisine, "Florentina puella ». Ainsi non seulement Étherius, mais toute la belle-famille d'Ursule avait trouvé la mort avec elle ${ }^{43}$...

On serait tenté de dire " et ainsi de suite " et nous renvoyons directement au texte des Revelationes édité par Roth le lecteur curieux de savoir qui était "saint Marcule évêque de Grèce " ou "sainte Gerasme ", ou encore quels furent les véritables auteurs du massacre des Onze mille vierges et qui se chargea de leur sépulture.

Pour toutes les questions qu'elle a à résoudre, Élisabeth procède de la même manière, déduisant de la juxtaposition de pierres tombales un lien, de parenté ou autre, entre les personnages dont elles abritent les restes et que la visionnaire de Schönau dote d'une biographie plus ou moins développée : ainsi connut-on tout d'un coup, dans leurs moindres détails, l'existence, l'origine et le sort de ces vierges qui, avant Élisabeth, étaient des êtres sans histoire, dont les textes qui les mentionnaient n'expliquaient ni la vie ni la mort.

Quand Élisabeth meurt, le 18 juin 1164, l'ère des révélations n'est pas pour autant tout à fait close : la nécropole romaine continue d'être une mine apparemment inépuisable de reliques qui deviennent, sous la plume d'un auteur incertain - «anonyme " selon G. de Tervarent ${ }^{44}$, Hermann de Steinfeld d'après F.W.E. Roth ${ }^{45}$ - celles d'autant de personnages épisodiques venus grossir le cortège virginal ; les deux livres de ses "Révélations ${ }^{46}$, qu'on peut dater des années 1184-1187, reprennent celles d'Élisabeth et les amplifient, portant à 769 le nombre de noms et de personnages connus à la fin du XII siècle. Il semble toutefois que ces nouvelles " révélations " n'aient jamais passé les Alpes et qu'elles aient connu une diffusion bien inférieure à celles d'Élisabeth : localisée dans un premier temps au sud et à l'ouest de Cologne, la légende telle qu'Élisabeth l'avait élaborée a vu sa diffusion se superposer étroitement à la dispersion géographique des reliques et dès le XII siècle, un moine de Norvège, Theoderich de Nidarholm, témoigne de sa pénétration dans son pays ${ }^{47}$.

Les données fournies par Élisabeth furent certes remaniées, résumées, ou partiellement critiquées : si Vincent de Beauvais ${ }^{48}$ ou Jacques de Voragine citent tels quels qui les "évêques de Bretagne ", qui le baptême de la mère d'Étherius, que seule Élisabeth avait fait connaître, l'auteur de la Légende dorée ne cache pas que l'époque à laquelle la visionnaire avait fixée la passion d'Ursule lui semble très

43. Idem, pp. 128-129.
44. La légende.., op. cit., p. 29.
45. Die Visionen..., op. cit., p. 118.
46. Editées in Acta sanctorum, Oct., t. IX, pp. 173-201.
47. Cité par G. DE TERVARENT, op. cit., p. 120.
48. Vincent DE BEAUVAIS, Speculum historiale, XXI, cap. 40-43. 
suspecte : " Elles souffrirent l'an du Seigneur 238 », écrit-il ${ }^{49}$. " La supputation des époques, d'après l'opinion de quelques-uns, ne permet pas de penser que ces choses se soient passées alors. (...) Il vaut mieux croire que ce fut après Constantin, au moment où les Huns et les Goths exerçaient leurs ravages, que ce martyre eut lieu, c'est-àdire du temps de l'empereur Marcien (selon qu'on le lit dans une chronique) qui régna l'an du Seigneur 352."

Le cadre de cette étude ne nous permet pas de développer davantage le thème de la fortune de cette légende dont on trouve la trace, notamment dans l'art, de Lerida à Oslo : aux derniers siècles du Moyen Age, l'image se substitue à la relique dans la diffusion du culte et avec l'iconographie, selon André Vauchez ${ }^{\text {so }}$, une nouvelle conception de la piété se fait jour, moins axée sur les restes des saints que sur leur existence terrestre. Notons toutefois que dans le cas qui nous intéresse, le trafic de reliques se poursuivit - en raison bien sûr de leur grand nombre - bien au-delà de l'époque de leur découverte, puisque Boniface IX, en 1381, dut interdire toute translation des reliques des Onze mille vierges et qu'il est probable qu'elles continuèrent néanmoins à circuler "sous le manteau "s! .

Tentons de conclure : l'exemple des Onze mille vierges nous semble certes un cas limite de l'histoire des reliques au Moyen Age, dont il illustre pleinement le caractère paradoxal : preuve de la mort, et donc signe a posteriori de l'existence de personnages réputés saints, la relique est aussi le garant de leur longévité, de leur survie dans la mémoire des hommes. La découverte fortuite de centaines d'ossements à Cologne au début du XII siècle permit aux vagues fantômes qui hantaient jusqu'alors une légende locale aux contours mal définis d'acquérir le statut de saintes personnes ; mais le hasard des excavations n'aurait pas suffi à assurer la célébrité d'une troupe de martyrs : une large part du succès de la Légende des Onze mille vierges au Moyen Age revient à l'habileté du clergé de Cologne, une autre au génie d'une jeune mystique influençable à qui ses révélations valurent d'être nommée supérieure du couvent féminin de Schönau en 1157. Elle mourut toutefois sans avoir épuisé le sujet et gageons que, si elle avait survécu, elle aurait été sollicitée à propos d'autres reliques : le 23 juillet 1164 selon F.W.E. Roth, un mois après la mort édifiante d'Élisabeth, l'archevêque Rainald fit en effet transférer de Milan à Cologne les reliques des rois mages ${ }^{52}$, et le monastère de Schönau en reçut une partie ${ }^{53}$.

49. La légende dorée, op. cit., t. II, p. 298.

50. La sainteté en Occident aux derniers siècles du Moyen Age, op. cit., p. 529.

51. G. DE Tervarent, op. cit., p. 21.

52. Épisode daté de 1162 dans les "Annales Sancti Disibodi" (MGH, loc. cit., p. 30) : "Reinoldus coloniensis episcopus corpora trium magorum (...) secum ad Coloniam singulis in locis transtulit (...)."

53. La demande en avait été formulée du vivant d'Élisabeth ; cf. F.W.E. Roth, op. cit., p. 112. 